\title{
Significantly decreased islet $\beta$ cell function and increased fasting plasma glucose in patients with chronic hepatitis B: a cross-sectional study
}

\section{Dafeng Liu ( $\triangle$ ldf312@126.com )}

Sichuan University West China Hospital, The Public and Health Centre of Chengdu https://orcid.org/00000002-6792-641X

\section{Lingyun Zhou}

Sichuan University West China Hospital

\section{Xinyi Zhag}

Sichuan University West China Medical Center

\section{Yilan Zeng}

The Public and Health Clinic Center of Chengdu

\section{Lang Bai}

Sichuan University West China Hospital

\section{Dongbo Wu}

Sichuan University West China Hospital

\section{Hong Tang}

Sichuan University West China Hospital

\section{Research Article}

Keywords: chronic hepatitis $B$, abnormal glucose metabolism, hemostasis model assessment of $\beta$ cells (HOMA- $\beta$ ), fasting plasma glucose, liver cirrhosis, hepatitis B envelope antigen ( $\mathrm{HBeAg})$

Posted Date: February 19th, 2021

DOI: https://doi.org/10.21203/rs.3.rs-146248/v2

License: (a) (i) This work is licensed under a Creative Commons Attribution 4.0 International License. Read Full License 


\section{Abstract}

Background: The contributing factors of abnormal glucose metabolism and the characteristics of the homeostasis model assessment of $\beta$ cell function (HOMA- $\beta$ ) value in chronic hepatitis $B(C H B)$ patients are unclear and worth studying.

Method: This cross-sectional study recruited $110 \mathrm{CHB}$ patients (CHB group) and 110 patients without hepatitis B virus (non-HBV group); the groups were matched according to sex, age, and body mass index. The contributing factors of abnormal glucose metabolism and the characteristics and differences in glucose metabolism parameters between the two groups were analyzed.

Results: The abnormal glucose metabolism rate was higher in CHB patients with liver cirrhosis (LC) and patients with hepatitis B envelope antigen ( $\mathrm{HBeAg})(-)$ status. In addition, under the same glucose metabolism conditions, the fasting plasma glucose (FPG) levels of the CHB group was higher than that of the non-HBV group, especially in those with LC that had higher FPG levels (all $p=0.000$ ), while the HOMA- $\beta$ values was significantly lower in the CHB group than in the non-HBV group, especially under normal glucose tolerance conditions (all $p=0.000$ ). Further analyses revealed that the main contributing factors of abnormal glucose metabolism were HBeAg (-) status and hepatitis B envelope antibody levels, but HBV serological and virological indicators had no direct effect on the HOMA- $\beta$ value.

Conclusion: These findings provide a reference that will allow clinicians to monitor abnormal glucose metabolism in CHB patients, especially those with LC or HBeAg (-) status, focus on the protection of islet $\beta-$ cell function, and avoid the application of insulin secretagogues in CHB patients with abnormal glucose metabolism.

\section{Introduction}

Approximately 300 million people worldwide are diagnosed with chronic hepatitis B (CHB), ${ }^{1}$ of whom approximately 650,000 die of hepatic failure, liver cirrhosis (LC), and hepatocellular carcinoma (HCC) annually. ${ }^{2}$ China is one of the HBV middle- and low-endemic areas worldwide, and approximately 93 million people are infected with $\mathrm{HBV}$; among them, 20 million present with $\mathrm{CHB} .{ }^{1,3}$

Although the relationship between hepatitis $B$ virus infection and diabetes mellitus (DM) remains controversial, several studies have shown that the prevalence rate of DM is significantly higher in the HBVinfected population, ${ }^{4-11}$ particularly in those with high viral load, with a long duration of $\mathrm{CHB}$, with cirrhosis, ${ }^{4,6,7-10}$ or of Asian American race. ${ }^{8}$ Our previous study has shown that $59.09 \%$ of CHB patients had high homeostasis model assessment of insulin resistance (HOMA-IR) values and $93.64 \%$ of individuals had low homeostasis model assessment of $\beta$ cell function (HOMA- $\beta$ ) values. Moreover, $35.45 \%$ of individuals had impaired glucose tolerance (IGT), ${ }^{12-13}$ which indicated that the prevalence rates of abnormal glucose metabolism and insulin resistance (IR) were high in patients with $\mathrm{CHB}$.

IR and DM could promote the progression of liver fibrosis and cirrhosis. ${ }^{14-17}$ Moreover, IR is independently correlated with the degree of liver fibrosis in patients with abnormal glutamyl transferase levels. ${ }^{12,14}$ The 
higher the HOMA-IR values, the higher the liver stiffness measurement (LSM) levels, an indicator of liver fibrosis. ${ }^{13,15}$ For DM patients with cirrhosis, the leading cause of death is hepatic failure as opposed to complications of DM. Furthermore, DM could also promote HCC and lead to poorer prognosis after liver transplantation $^{18,19}$. Therefore, the coexistence of abnormal glucose metabolism and IR could promote the progression and worsen the prognosis of $\mathrm{CHB}$.

Abnormal glucose metabolism and IR are commonly associated with islet $\beta$ cell dysfunction in the general population, particularly in Asian populations. However, in patients with $\mathrm{CHB}$, the contributing factors of glucose abnormalities and the characteristics associated with HOMA- $\beta$ values are unclear and worth further study.

\section{Methods}

\section{Study population}

A cross-sectional study with a sample size of 220 patients was conducted in the Public and Health Clinic Centre of Chengdu from January 1, 2012, to June 30, 2013. Among them, 110 patients with CHB were assigned to the CHB group (the source of the cases has been previously explained in the literature). ${ }^{12-13}$ One hundred ten patients without hepatitis B virus, hepatitis $\mathrm{C}$ virus (HCV) and human immunodeficiency virus (HIV) infection who were matched to the CHB group according to sex, age, and body mass index (BMI) were assigned to the non-HBV group. The study was approved by the ethics committee of the Public and Health Clinic Centre of Chengdu (PJ-K2019-019-01). All patients provided written informed consent.

The inclusion criteria of the CHB group were as follows: (1) outpatients or inpatients with CHB or posthepatitis B cirrhosis; (2) individuals who agreed to undergo noninvasive ultrasound liver stiffness measurement; and (3) individuals aged 18-70 years.

The selection criteria of the non-HBV group were as follows: patients without hepatitis B virus infection, matched to the CHB group according to sex, age and BMI and time.

The following exclusion criteria were used in this study: (1) other hepatitis virus or human immunodeficiency virus infection; (2) hepatocellular carcinoma; (3) ascites; (4) decompensated cirrhosis; (5) hepatic function alanine aminotransferase (ALT) or aspartate aminotransferase (AST) level higher than the 5-fold upper limit of the normal value, total bilirubin level higher than the 2-fold upper limit of the normal value within the last 6 months, or prothrombin activity (PT\%) <60\%; and (6) BMI $>30 \mathrm{~kg} / \mathrm{m}^{2}$ or $<18.5 \mathrm{~kg} / \mathrm{m}^{2}$.

The diagnostic criteria of the diseases were as follows: CHB diagnostic and typing criteria, impaired glucose regulation (IGR) and DM diagnostic criteria were applied according to the corresponding guidelines. ${ }^{20,21}$

The participants were divided into three subgroups according to their glucose metabolism conditions: the normal glucose tolerance (NGT) condition group [fasting plasma glucose (FPG) levels $<6.0 \mathrm{mmol} / \mathrm{L}$ and $2-$ hour postprandial glucose $(2 \mathrm{hPG}$ ) levels $<7.8 \mathrm{mmol} / \mathrm{L}]$, the IGR condition group (FPG and $2 \mathrm{hPG}$ levels all 
between those in the NGT and DM condition), and the DM condition group (FPG levels $\geq 7.0 \mathrm{mmol} / \mathrm{L}$ and $2 \mathrm{hPG}$ levels $\geq 11.1 \mathrm{mmol} / \mathrm{L}$ or twice that of the $\mathrm{FPG}$ or $2 \mathrm{hPG}$ levels meeting the criteria).

The participants in the CHB group were also divided into LC and non-LC subgroups based on the presence or absence of cirrhosis, respectively, and hepatitis B envelope antigen ( $\mathrm{HBeAg})(-)$ and $\mathrm{HBeAg}(+)$ subgroups according to HBeAg negative or positive status, respectively.

\section{Clinical data collection}

Demographic information (age and sex), anthropometric parameters (body weight and height), glucose metabolic parameters [FPG levels, 2 hPG levels, fasting insulin (FINS) levels, 2-h postprandial insulin (2hINS) levels, and hemoglobin A1c (HbA1c) levels] were obtained. BMI, HOMA-IR values, and HOMA- $\beta$ values were calculated by using the following formulas: $\mathrm{BMI}=$ weight $(\mathrm{kg}) / \mathrm{height}\left(\mathrm{m}^{2}\right), \mathrm{HOMA}-\mathrm{IR}=$

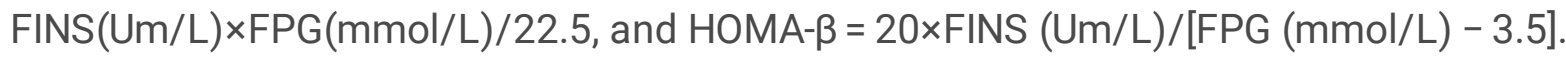

Databases were established according to the needs of the research. Two researchers simultaneously collected and entered the data into the database. Then, the researchers randomly selected $30 \%$ of the data for assessment to ensure data integrity, authenticity, and accuracy.

\section{Statistical analyses}

The Statistical Package for the Social Sciences software version 17.0 (IBM Inc. Armonk, NY, USA) and Prism Version 8 (GraphPad Inc. US) were used for statistical analysis. Age, BMI, FPG and FINS levels, and HOMA-IR values had a normal distribution, and the statistical analysis was conducted directly. Natural HOMA- $\beta$ values were logarithmically transformed before the statistical analysis. The measurement data were expressed as $\mathrm{x}$ $\pm S D$, ANOVA was used for a multigroup comparison with variance homogeneity and normal distribution data, and the least significant difference (LSD) $t$ test was used for further comparison between the two groups. For variables without variance homogeneity and normally distributed data, the independent sample Kruskal-Wallis $\mathrm{H}(\mathrm{K})$ test was used for multigroup comparisons, and the Mann-Whitney Utest was used for further comparisons between the two groups. Independent-sample $t$-tests were compared between two groups. The percentage or proportion was expressed for enumeration data, and the chi-square test was used for comparison of these data. Spearman correlation analysis was adopted for two-factor correlation analysis, and multiple stepwise regression was applied for multifactor correlation analysis. Statistical significance was defined as $P<0.05$.

\section{Results}

\section{Patients}

We enrolled a total of 220 patients, among whom 110 were assigned to the CHB group and 110 were assigned to the non-HBV group. No significant differences were observed in terms of age, sex, BMI or glucose metabolism conditions between the two groups. (Table 1). For the CHB group, 41 (37.27\%) patients had LC, and 38 (34.55\%) patients were HBeAg(+). In the non-HBV group, there were no cases of LC. 
Table 1

Baseline comparison between the two groups $(n=220)$

\begin{tabular}{|c|c|c|c|c|}
\hline Variables & $\begin{array}{l}\text { CHB group }(n= \\
110)\end{array}$ & $\begin{array}{l}\text { Non-HBV group }(n= \\
110)\end{array}$ & $\begin{array}{l}t \text { score or } \chi^{2} \\
\text { score }\end{array}$ & $\begin{array}{l}P \\
\text { score }\end{array}$ \\
\hline Age (years) & $43.86 \pm 14.38$ & $42.68 \pm 13.34$ & $t=0.794$ & 0.428 \\
\hline Male (number, \%) & $90(81.92 \%)$ & $90(81.92 \%)$ & $x^{2}=0.000$ & 1.000 \\
\hline BMI $\left(\mathrm{kg} / \mathrm{m}^{2}\right)$ & $22.52 \pm 2.74$ & $23.14 \pm 4.07$ & $t=-1.245$ & 0.215 \\
\hline $\begin{array}{l}\text { Glucose metabolism } \\
\text { conditions }\end{array}$ & & & $x^{2}=0.000$ & 1.000 \\
\hline NGT & $50(45.46 \%)$ & $50(45.46 \%)$ & & \\
\hline IGR & $30(27.27 \%)$ & $30(27.27 \%)$ & & \\
\hline DM & $30(27.27 \%)$ & $30(27.27 \%)$ & & \\
\hline
\end{tabular}

Abbreviations: $\mathrm{CHB}$, chronic hepatitis $\mathrm{B}$; non-HBV, without hepatitis $\mathrm{B}$ virus infection; BMI, body mass index; NGT, normal glucose tolerance; IGR, impaired glucose regulation; DM, diabetes mellitus.

\section{Abnormal glucose metabolism rate}

The IGR and DM rates in the CHB group were all 27.27\% (30/110). In addition, the abnormal glucose metabolism rate (including IGR and DM) of the LC subgroup was significantly higher than that of the non-LC subgroup (NGT, IGR, DM: $19.51 \%, 43.90 \%, 36.59 \%$ vs. $60.87 \%, 17.39 \%, 21.74 \%, \chi^{2}=3.588, P=0.000$ ). Additionally, the glucose abnormality rate in the HBeAg (-) subgroup was also significantly higher than that in the HBeAg (+) subgroup ((NGT, IGR, DM: 33.33\%, 31.94\%, 34.72\%vs. $68.42 \%, 18.42 \%, 13.16 \%, \chi^{2}=6.174, P$ $=0.000)$.

\section{HOMA- $\beta$ value and FPG level}

Under NGT conditions in the CHB group, the FPG levels (Fig. 1A) was slightly higher and the FINS levels (Fig. 1B), the HOMA-IR values (Fig. 1C), and the HOMA- $\beta$ values (Fig. 1D) were all significantly lower than those of the non-HBV group ( $P=0.019,0.000,0.000,0.000$, respectively).

Under NGT conditions, the HOMA- $\beta$ values of the non-HBV group was $124.19 \mathrm{mlU} / \mathrm{mmol}$ (reference values: $100.00 \mathrm{mlU} / \mathrm{mmol})$, while in the CHB group, it was only $47.53 \mathrm{mlU} / \mathrm{mmol}$. Compared with the non-HBV group and reference values, the decreased amplitudes of the HOMA- $\beta$ values in the CHB group were 77.79 $\mathrm{mlU} / \mathrm{mmol}$ and $53.47 \mathrm{mlU} / \mathrm{mmol}$, respectively, and the reduction percentages of the HOMA- $\beta$ values in the CHB group were $62.64 \%$ and $53.47 \%$, respectively.

With the deterioration of glucose metabolism from the NGT and IGR conditions to the DM condition, FPG levels (Fig. 2A), FINS levels (Fig. 2B) and HOMA-IR values (Fig. 2C) continuously increased, while HOMA- $\beta$ values (Fig. 2D) continuously decreased in the two groups (all $P=0.000$ ). 
Moreover, $\mathrm{CHB}$ patients always had similar changes in glucose parameters under IGR and DM conditions as well as under NGT conditions: in the CHB group, the FPG levels (Fig. 2A) was higher and the FINS levels

(Fig. 2B), the HOMA-IR values (Fig. 2C) and the HOMA- $\beta$ values (Fig. 2D) were all lower than those of the nonHBV group under the same glucose metabolism conditions $(P=0.000,0.000,0.0024,0.000$, respectively).

For the non-HBV group, under IGR and DM conditions, the FPG levels were all lower than $6.0 \mathrm{mmol} / \mathrm{L}$, while in the $\mathrm{CHB}$ group, the FPG levels were more than $6.0 \mathrm{mmol} / \mathrm{L}$ under IGR conditions and more than $7.0 \mathrm{mmol} / \mathrm{L}$ under DM conditions.

\section{The change characteristics of glucose metabolism parameters}

With the deterioration of glucose metabolism from the NGT and IGR conditions to the DM condition, the FPG levels (Fig. 3A), the HOMA-IR values (Fig. 3C), and the FINS levels (Fig. 3B) were all continuously increased $(P=0.000,0.000,0.0185$, respectively), while the HOMA- $\beta$ values (Fig. 3D) were all continuously decreased $(P=0.000)$ in both the LC and non-LC subgroups.

In the LC subgroup, the HOMA- $\beta$ values (Fig. 3D) was higher under NGT conditions but significantly lower under IGR and DM conditions than in the non-LC subgroup. At the same time, the FPG levels (Fig. 3A) was higher under NGT and IGR conditions than in the non-LC subgroup. However, there were no significant differences in the FINS levels (Fig. 3B) or the HOMA-IR values (Fig. 3C) under the same glucose metabolism conditions or in the FPG levels under DM conditions between the LC and non-LC subgroups (all $P>0.05$ ).

With the deterioration of glucose metabolism from the NGT and IGR conditions to DM conditions, the FPG levels (Fig. 4A), FINS levels (Fig. 4B) and HOMA-IR values (Fig. 4C) of the two subgroups were all continuously increased $(P=0.000,0.0055,0.000)$, while the HOMA- $\beta$ values (Fig. 4D) were all continuously decreased $(P=0.0031)$ in both the HBeAg $(+)$ and HBeAg $(-)$ subgroups.

Similar changes in all glucose parameters (Fig. 4A, 4B, 4C and 4D) could be seen in CHB patients who were HBeAg (+) and (-), and there was no significant difference between the two subgroups under the same glucose metabolism conditions (all $P>0.05$ ).

\section{The contributing factors of glucose metabolism}

According to Spearman correlation analysis, LC, hepatitis B envelope antibody (HBeAb) levels, alkaline phosphatase (ALP) levels, gamma glutamyl transferase (GGT) levels, and liver stiffness measurement (LSM) levels were positively correlated, but HBeAg (+), hepatitis B surface antigen (HBsAg) levels, HBeAg levels, hepatitis $B$ core antibody (HBcAb) levels, and hepatitis $B$ viral nucleic acid (HBVDNA) levels were all negatively correlated with glucose metabolism condition (Table 2). In addition, HBeAb levels, ALP levels, GGT levels, and LSM levels were also positively correlated, while HBeAg (+), HBsAg levels, HBeAg levels, and HBVDNA levels were all negatively correlated with FPG levels (Table 2). Based on multiple stepwise regression analysis, $\mathrm{HBeAg}(-), \mathrm{GGT}$ levels and HBeAb levels were the contributing factors for glucose metabolism, and HBeAb levels was the contributing factor for FPG levels (Table 3). 
Table 2

Spearman correlation analysis between glucose metabolism parameters and HBV-related biological and serum parameters $(n=110)$

\begin{tabular}{|c|c|c|c|c|c|c|c|c|c|c|}
\hline \multirow[t]{2}{*}{ Variable } & \multicolumn{2}{|c|}{$\begin{array}{l}\text { Glucose } \\
\text { metabolism } \\
(1=\text { NGT, } 2= \\
\text { IGR, } 3=\text { DM) }\end{array}$} & \multicolumn{2}{|c|}{$\begin{array}{l}\text { FPG } \\
(\mathrm{mmol} / \mathrm{L})\end{array}$} & \multicolumn{2}{|c|}{$\begin{array}{l}\text { FINS } \\
(\mathrm{mU} / \mathrm{L})\end{array}$} & \multicolumn{2}{|c|}{$\begin{array}{l}\text { HOMA-IR } \\
\left(\mathrm{mU} * \mathrm{mmol} / \mathrm{L}^{2}\right)\end{array}$} & \multicolumn{2}{|c|}{$\begin{array}{l}\text { HOMA- } \beta \\
(\mathrm{mU} / \mathrm{mmol})\end{array}$} \\
\hline & $r$ & p & $r$ & p & $r$ & $\mathbf{p}$ & $r$ & p & $r$ & p \\
\hline $\begin{array}{l}\text { Cirrhosis(1 } \\
= \\
\text { without,2 } \\
=\text { with) }\end{array}$ & 0.191 & 0.046 & & & 0.322 & 0.001 & 0.328 & 0.000 & & \\
\hline $\begin{array}{l}\text { HBeAg (1 } \\
=\text { negative, } \\
2= \\
\text { positive) }\end{array}$ & -0.330 & 0.000 & -0.268 & 0.005 & & & & & & \\
\hline $\mathrm{HBsAg}$ & -0.350 & 0.000 & -0.334 & 0.000 & & & & & 0.200 & 0.036 \\
\hline HBsAb & & & & & -0.270 & 0.004 & -0.199 & 0.037 & -0.281 & 0.003 \\
\hline $\mathrm{HBeAg}$ & -0.321 & 0.001 & -0.232 & 0.015 & & & & & & \\
\hline HBeAb & 0.396 & 0.000 & 0.333 & 0.000 & & & 0.240 & 0.012 & & \\
\hline $\mathrm{HBcAb}$ & -0.205 & 0.032 & & & & & & & & \\
\hline HBVDNA & -0.202 & 0.034 & -0.190 & 0.047 & & & & & & \\
\hline ALP & 0.247 & 0.009 & 0.286 & 0.002 & & & & & -0.361 & 0.000 \\
\hline GGT & 0.354 & 0.000 & 0.293 & 0.002 & & & & & & \\
\hline LSM & 0.260 & 0.006 & 0.272 & 0.004 & 0.230 & 0.015 & 0.306 & 0.000 & & \\
\hline \multicolumn{11}{|c|}{$\begin{array}{l}\text { Abbreviations: ALP, alkaline phosphatase. FINS, fasting serum insulin. DM, diabetes mellitus. FPG, } \\
\text { fasting plasma glucose. GGT, gamma glutamyl transferase. HbA1c, glycosylated hemoglobin. HBcAb, } \\
\text { hepatitis B core antibody. HBeAb, hepatitis B envelope antibody. HBeAg, hepatitis B envelope antigen. } \\
\text { HBsAb, hepatitis B surface antibody. HBsAg, hepatitis B surface antigen. HBVDNA, hepatitis B viral } \\
\text { nucleic acid load. HOMA- } \beta \text {, homeostasis model assessment of } \beta \text { cells. HOMA-IR, homeostasis model } \\
\text { assessment of insulin resistance. IGR, impaired glucose regulation. LSM, liver stiffness measurement. } \\
\text { NGT, normal glucose tolerance. }\end{array}$} \\
\hline
\end{tabular}


Table 3

Multiple stepwise regression analysis of influencing factors of HBV-related biological and serum parameters on glucose metabolism parameters $(n=110)$

\begin{tabular}{|c|c|c|c|c|c|c|}
\hline Independent variable & & B & $\begin{array}{l}\text { Std. } \\
\text { Error }\end{array}$ & Beta & $\mathrm{t}$ & p \\
\hline \multirow{4}{*}{$\begin{array}{l}\text { Glucose metabolism } \\
(1=\text { NGT, } 2=I G R, 3= \\
\text { DM })\end{array}$} & constant & 0.730 & 0.273 & - & 2.678 & 0.009 \\
\hline & $\begin{array}{l}\mathrm{HBeAg}(1=\text { negative, } 2= \\
\text { positive) }\end{array}$ & -0.430 & 0.161 & -0.249 & -2.663 & 0.009 \\
\hline & GGT & 0.002 & 0.001 & 0.298 & 3.291 & 0.001 \\
\hline & $\mathrm{HBeAb}$ & 0.117 & 0.045 & 0.242 & 2.600 & 0.011 \\
\hline \multirow{2}{*}{$\begin{array}{l}\text { FPG } \\
(\mathrm{mmol} / \mathrm{L})\end{array}$} & constant & 5.463 & 0.266 & - & 20.530 & 0.000 \\
\hline & $\mathrm{HBeAb}$ & 0.494 & 0.126 & 0.378 & 3.933 & 0.000 \\
\hline \multirow{2}{*}{$\begin{array}{l}\text { FINS } \\
(\mathrm{mU} / \mathrm{L})\end{array}$} & constant & 3.069 & 0.787 & - & 3.900 & 0.000 \\
\hline & $\begin{array}{l}\text { Cirrhosis ( } 1 \text { = without, } 2 \text { = } \\
\text { with) }\end{array}$ & 1.123 & 0.531 & 0.214 & 2.117 & 0.037 \\
\hline \multirow{3}{*}{$\begin{array}{l}\text { HOMA-IR } \\
\left(\mathrm{mU}^{\star} \mathrm{mmol} / \mathrm{L} 2\right)\end{array}$} & constant & 0.560 & 0.269 & - & 2.081 & 0.040 \\
\hline & $\mathrm{HBeAb}$ & 0.133 & 0.054 & 0.225 & 2.453 & 0.016 \\
\hline & $\begin{array}{l}\text { Cirrhosis }(1=\text { without, } 2 \text { = } \\
\text { with) }\end{array}$ & 0.426 & 0.180 & 0.216 & 2.358 & 0.020 \\
\hline \multirow{2}{*}{$\begin{array}{l}\text { HOMA- } \beta \\
(\mathrm{mU} / \mathrm{mmol})\end{array}$} & constant & 66.452 & 8.387 & - & 7.923 & 0.000 \\
\hline & ALP & -0.183 & 0.074 & -0.230 & -2.459 & 0.016 \\
\hline \multicolumn{7}{|c|}{$\begin{array}{l}\text { Abbreviations: ALP, alkaline phosphatase. FINS, fasting serum insulin. DM, diabetes mellitus. FPG, } \\
\text { fasting plasma glucose. GGT, gamma glutamyl transferase. HBeAb, hepatitis B envelope antibody. } \\
\text { HBeAg, hepatitis B envelope antigen. HOMA- } \beta \text {, homeostasis model assessment of } \beta \text { cells. HOMA-IR, } \\
\text { homeostasis model assessment of insulin resistance. IGR impaired glucose regulation. NGT, normal } \\
\text { glucose tolerance. }\end{array}$} \\
\hline
\end{tabular}

According to Spearman correlation analysis, LC and LSM levels were positively correlated with FINS levels; simultaneously, LC, LSM levels and HBeAb levels were also positively correlated with HOMA-IR values, while hepatitis B surface antibody (HBsAb) levels were negatively correlated with both FINS levels and HOMA-IR values (Table 2). Based on multiple stepwise regression analysis, LC was a contributing factor for both FINS levels and HOMA-IR values (Table 3).

Only the HBsAg levels was positively correlated, while the HBsAb levels and ALP levels were negatively correlated with HOMA- $\beta$ values, but only the ALP levels was a contributing factor for HOMA- $\beta$ values (Table 3).

\section{Discussion}


This study revealed that the prevalence rate of both IFG and DM was $27.27 \%$ in patients with HBV infection, and the abnormal glucose metabolism rate was higher in CHB patients with LC or HBeAg (-) status.

Some of the findings in this study were similar to those in previous literature, which reported that the prevalence of DM was significantly higher in the HBV-infected population, ${ }^{4-11}$ particularly in those with high viral load, with a long duration of $\mathrm{CHB}$, with cirrhosis, ${ }^{4,6,7-10}$ or of Asian American race. ${ }^{8}$ A meta-analysis reported that the summary OR of the risk of DM for HBV patients was $1.99(95 \% \mathrm{Cl}, 1.08-3.65)$ when compared with non-HBV individuals. ${ }^{4}$ The prevalence rates of both IFG and DM in this study were higher than the $12.5 \%$ for DM and $7.8 \%$ for IFG in adults with CHB previously reported in a large HBV-infected multiethnic cohort study. ${ }^{8}$ This might be attributed to the differences in the study population: the populations of the two former studies were Chinese, while the population of the latter study was American.

This study also reported a higher abnormal glucose metabolism (including IGR and DM) rate in CHB patients with LC. The prevalence rates of IGR and DM were $43.90 \%$ and $36.59 \%$ in CHB patients with LC in comparison to $18.42 \%$ and $13.16 \%$ in CHB patients without LC. This finding is consistent with those from a study that reported ${ }^{7-10,17}$ that the odds ratios for $D M$ in chronic hepatitis $B$ cirrhosis patients were $1.74,1.76$ and 2.317 (95\% confidence interval: $1.43-2.13,1.44-2.14,1.528-3.513$, respectively) when compared with noncirrhotic chronic hepatitis B patients. ${ }^{7-10,17}$ However, the DM prevalence was higher than in other crosssectional studies reporting a prevalence of $22.2 \%$ of DM among CHB patients with liver cirrhosis. ${ }^{17}$ The development of cirrhosis may increase the incidence of $\mathrm{DM} .{ }^{7-10,17}$

In addition, it is proposed that this is the first study to indicate that the abnormal glucose metabolism rate was higher in CHB patients who were HBeAg negative, with IGR and DM prevalence rates of $31.94 \%$ and $34.72 \%$ in patients who were HBeAg negative in comparison to $17.39 \%$ and $21.74 \%$ in patients who were HBeAg positive. Previous studies suggested that the HBsAg status could influence glucose metabolism, and maternal HBsAg carriers were an independent risk factor for gestational diabetes mellitus (GDM). ${ }^{22}$ The incidence of GDM in pregnant women who were HBsAg positive was $6.48 \%$, which was higher than the $3.41 \%$ incidence rate in those who were HBsAg negative. ${ }^{23}$ However, there was no significant association between the incidence of DM and viral load, HBeAg carrier status, or other HBV markers in pregnancy, ${ }^{22-23}$ and there is no literature reporting glucose metabolism in HBeAg carriers.

Further analyses demonstrated that abnormal glucose metabolism manifested as elevated FPG levels and significantly decreased islet $\beta$ cell function, as indicated by the HOMA- $\beta$ values, but it did not manifest as insulin resistance, as indicated by the HOMA-IR values. We found that under the same glucose metabolism conditions, the FPG level of the CHB group was continuously higher than that of the non-HBV group; it was more than $6.0 \mathrm{mmol} / \mathrm{L}$ under IGR conditions and more than $7.0 \mathrm{mmol} / \mathrm{L}$ under DM conditions, while in the non-HBV group, it was lower than $6.0 \mathrm{mmol} / \mathrm{L}$ under all three glucose metabolism conditions.

Simultaneously, the HOMA-IR value of the CHB group was consistently lower than that of the non-HBV group. However, under NGT conditions, the HOMA- $\beta$ values of the HBV group was $47.53 \mathrm{mlU} / \mathrm{mmol}$, only half of the reference value $(100.00 \mathrm{mlU} / \mathrm{mmol})$, and only one third of the non-HBV group $124.19 \mathrm{mlU} / \mathrm{mmol}$, and these values continuously decreased with the deterioration of glucose metabolism. 
A multicenter randomized parallel-group trial showed that the HOMA- $\beta$ values in patients newly diagnosed with DM was only half the reference value $\left(100 \mathrm{mmol}{ }^{*} \mathrm{mIU} / \mathrm{L}^{2}\right)$; it decreased progressively at a rate of $4.5 \%$ annually and deteriorated with the course of the disease. ${ }^{24}$ A new staging method for NGT, IGR and DM was proposed according to the function of $\beta$ cells: normal phase of $\beta$ cell function, compensatory phase of $\beta$ cell function, decompensatory phase of $\beta$ cell function, and failure phase of $\beta$ cell function in the general population. The compensatory secretion of $\beta$ cell function occurs in individuals with NGT and IR and reaches the peak of compensatory secretion. The decompensatory phase of $\beta$ cell function has occurred in individuals with prediabetes or IGR. ${ }^{25}$ In recent years, most studies have confirmed that not all individuals with NGT were healthy, and some presented with IR. ${ }^{26}$ The risk of developing prediabetes and/or DM significantly increased in individuals with NGT but IR and dysfunction of $\beta$ cells. ${ }^{26}$ Therefore, the HOMA- $\beta$ values of CHB patients under NGT conditions was even lower than that of those newly diagnosed with DM. The $\beta$ cell function of the $\mathrm{CHB}$ population deteriorates directly to the decompensatory and failure phases, without undergoing normal and compensatory phases, even under NGT conditions, and this change leads to higher FPG levels and a high prevalence of IGR and DM in the CHB population. From this, we could conclude that the evident increase in the FPG levels in patients with CHB was associated with worsening $\beta$ cell function but not insulin resistance.

In this study, it was also demonstrated that most of the HBV serological and virological indicators had negative effects, while LC, HBeAb levels and markers of liver inflammation and fibrosis had positive effects on both glucose metabolism and FPG levels. The main contributing factors for glucose metabolism and FPG levels were $\mathrm{HBeAg}(-)$ and HBeAb levels. However, HBV serological and virological indicators had no direct effects on islet $\beta$ cell function, as indicated by the HOMA- $\beta$ values. Therefore, we speculated that HBV indirectly affected islet $\beta$ cell function through certain mechanisms.

Fundamental studies have found that hepatitis B virus infection could increase the production of tumor necrosis factor (TNF), especially in HBeAg-negative patients. ${ }^{27}$ The overproduction of TNF could decrease the phosphorylation of insulin receptor substrates 1 and 2, inhibit phosphoinositol 3-kinase and protein kinase $B$, block the phosphorylation of glucose transporter 4, prevent the cell uptake of glucose ${ }^{28}$ and increase plasma glucose levels. Prostate six-transmembrane protein 2 (STAMP2) is a factor associated with inflammation and dietary adipocyte function and system metabolism. It can be induced by nutrition, feeding, and cytokines, such as TNF alpha, interleukin (IL)-1 3 , and IL-6, which can inhibit IR in rats. IR and visceral and hepatic insulin signaling disorders were observed in mice lacking STAMP2. In the presence of inflammation and obesity, the increased expression of STAMP2 has protective effects against insulin signaling in the liver. ${ }^{29}$ Moreover, hepatitis B virus X protein induces liver fat accumulation and IR by reducing the expression of STAMP2. STAMP2 downregulates the insulin-induced phosphorylation of the P3K p85 subunit, protein kinase and the expression of insulin receptor substrate 1, and the posttranscriptional level of insulin receptor substrate 1 plays a role, ${ }^{30}$ this leads to the increase in blood glucose levels and high abnormal glucose metabolism incidence.

Although those fundamental science studies have confirmed that hepatitis B virus infection could lead to increased hepatic glucose output and IR, they could not explain the decrease in HOMA- $\beta$ values and FINS 
levels. Further fundamental science studies are needed to investigate the mechanisms of the hepatitis $B$ virus infection effect on islet $\beta$ cell function in CHB patients.

To our knowledge, this cross-sectional study was the first to compare the differences in HOMA- $\beta$ values and FPG levels between CHB patients and non-HBV patients matched according to sex, age and BMI. This is the first study to compare the differences in abnormal glucose metabolism rates between CHB patients with HBeAg (+) and HBeAg (-) status, and it is also the first study to analyze the contributing factors of HBV virological and serological indicators on abnormal glucose metabolism. The results showed that in $\mathrm{CHB}$ patients, the FPG levels was higher, while the HOMA- $\beta$ values was significantly lower. Additionally, the HOMAIR values was also lower than that of non-HBV patients under the same glucose metabolism conditions. The $\beta$ cell function of the $\mathrm{CHB}$ population deteriorated directly to the decompensatory and failure phases, without undergoing normal and compensatory phases, even under NGT conditions. Furthermore, CHB patients with LC or who were HBeAg negative had a higher abnormal glucose metabolism rate. HBV serological and virological indicators, markers of liver inflammation and fibrosis, and LC could directly affect glucose metabolism. However, the effects on islet $\beta$ cell function were indirect. Therefore, the increased FPG levels of $\mathrm{CHB}$ patients was accompanied by significantly decreased islet $\beta$ cell function but not insulin resistance. In addition, HBV could directly affect glucose metabolism and could indirectly affect islet $\beta$ cell function through certain mechanisms.

Our study also has some limitations. The sample size was small, and it was a single-center, retrospective study. A further multicenter, prospective study with a large sample size is needed.

The findings of this study provide a reference to allow clinicians to monitor abnormal glucose metabolism in $\mathrm{CHB}$ patients, especially those with $\mathrm{LC}$ or $\mathrm{HBeAg}(-)$, focus on the protection of islet $\beta$-cell function, and avoid the application of insulin secretagogues in CHB patients with abnormal glucose metabolism.

\section{Declarations}

\section{Disclosure}

The authors declare that they have no competing interests.

\section{Funding}

This research was supported by the Sichuan Province Health Commission (17PJ070), Chengdu Municipal Health Commission (2019079), Natural Science Foundation of China (81802468) and Sichuan Science and Technology Program (2019YFS0207). The funders had no role in the study design, data collection and analysis, decision to publish, or preparation of the manuscript.

\section{Acknowledgments}


We would like to thank Drs. Rong Hu, Lin Wang, Li Wang and Zhu Chen (the Public and Health Clinic Centre of Chengdu, first ward, second ward, and third ward of liver disease department, respectively).

\section{References}

1. Polaris Observatory Collaborators, Global prevalence, treatment, and prevention of hepatitis $B$ virus infection in 2016: a modelling study. Lancet Gastroenterol Hepatol 3, 383-403 (2018)

2. R. Lozano, M. Naghavi, K. Foreman, S. Lim, K. Shibuya et al. Global and regional mortality from 235 causes of death for 20 age groups in 1990 and 2010: a systematic analysis for the Global Burden of Disease Study 2010. Lancet2012; 380:2095-2128

3. F.M. Lu, H. Zhuang, Management of hepatitis B in China. Chin Med J (Engl) 122, 3-4 (2009)

4. J. Zhang, Y. Shen, H. Cai, Y.M. Liu, G. Qin, Hepatitis B virus infection status and risk of type 2 diabetes mellitus: A meta-analysis. HepatolRes2015; 45:1100-1109

5. P.R. Spradling, B. Simons, M. Narayanan, J. Xing, C. Homan et al., Incidence of diabetes mellitus in population-based cohort of persons with chronic hepatitis B virus infection. J Viral Hepat 20,510-513 (2013)

6. Y. Shen, J. Zhang, H. Cai, J.G. Shao, Y.Y. Zhang et al., Identifying patients with chronic hepatitis B at high risk of type 2 diabetes mellitus: a cross-sectional study with pair matched controls. BMC Gastroenterol 15, 32 (2015)

7. Y. Shen, S. Zhang, X. Wang, Y. Wang, J. Zhang et al., Comparison of type 2 diabetes mellitus incidence in different phases of hepatitis B virus infection: A meta-analysis. Liver Int 37, 1451-1460 (2017)

8. M. Li-Ng, S. Tropp, A. Danoff, E.J. Bini, Association between chronic hepatitis B virus infection and diabetes among Asian Americans and Pacific Islanders. Dig Liver Dis 39, 549-556 (2007)

9. M. Khalili, M. Lombardero, R.T. Chung, N.A. Terrault, M.G. Ghany et al. Diabetes and prediabetes in patients with hepatitis B residing in North America. Hepatology2015; 62(5):1364-74

10. Y. Pang, C. Kartsonaki,2, I. Turnbull, Y. Guo, R. Clarke et al. Diabetes, Plasma Glucose, and Incidence of Fatty Liver, Cirrhosis, and Liver Cancer: A Prospective Study of 0.5 Million People. Hepatology2018; 68(4):1308-1318

11. Y. Liu, S. Ye, X. Xiao, T. Zhou, S. Yang et al., Association of diabetes mellitus with hepatitis B and hepatitis C virus infection: evidence from an epidemiological study. Infect Drug Resist 12, 2875-2883 (2019)

12. D.F. Liu, Y.L. Zeng, J. Lin, L.J. Lan, Y. Wang et al., The analysis of expression of the metabolism abnormal manifestation in patients with chronic hepatitis B. J Chengdu Med Coll (Chin) 9, 712-716 (2014)

13. D.F. Liu, Y.L. Liu, Y.L. Zeng, L. Wang, J. Lin et al., Effects of metabolic abnormalities on liver fibrosis in patients with chronic hepatitis B. Chin J Exp Clin Infect Dis (Electron Ed) (Chin) 10, 541-545 (2016)

14. S. Petta, F.S. Macaluso, M.R. Barcellona, C. Cammà, D. Cabibi et al., Serum Y-glutamyl transferase levels, insulin resistance and liver fibrosis in patients with chronic liver diseases. PLOS ONE 7, e51165 (2012) 
15. Y.W. Huang, T.C. Wang, S.C. Lin, H.Y. Chang, D.S. Chen et al., Increased risk of cirrhosis and its decompensation in chronic hepatitis $B$ patients with newly diagnosed diabetes: a nationwide cohort study. Clin Infect Dis 57, 1695-1702 (2013)

16. X. Li, Y. Jiao, Y. Xing, P. Gao. Diabetes Mellitus and Risk of Hepatic Fibrosis/Cirrhosis. Biomed Res Int $2019 ; 2019: 5308308$

17. J. XuC,Chen, ZhangPA. Relationship between diabetes mellitus and cirrhosis risk in chronic hepatitis $B$ patients in Wuhan, China. Med SciMonit 25, 8112-8119 (2019)

18. XuH. LiX, GaoP.Diabetes Mellitus is a Risk Factor for Hepatocellular Carcinoma in Patients with Chronic Hepatitis B Virus Infection in China. Med. Sci. Monit. 24, 6729-6734 (2018)

19. WeiS. TanY, YangJ. ZhangW, YangJ et al., Type 2 diabetes mellitus increases the risk of hepatocellular carcinomain subjects with chronic hepatitis B virus infection: a meta analysis and systematic review. Cancer Manag Res 11, 705-713 (2019)

20. Society of Hepatology, Chinese Medical Association. Society of Infectious Diseases, Chinese Medical Association. Guidelines for the Prevention and Treatment of Chronic Hepatitis B (2010) edition). Chin J Hep (Chin) 2011; 19:13-24

21. Diabetes Society of Chinese Medical Association, Guidelines for the Prevention and Treatment of Type 2 Diabetes in China (2010) edition). Chin J Diab (Chin) 20, S1-S37 (2012)

22. S.X. Peng, Z.H. Wan, X.F. Lin, X. Li, Y.K. Du, Maternal Hepatitis B Surface Antigen Carrier Status Increased the Incidence of Gestational Diabetes Mellitus. BMC Infect Dis 19(1), 147 (2019)

23. J. Tan, X.Y. Mao, G.T. Zhang, W. Wang, T.Y. Pan et al., Hepatitis B Surface Antigen Positivity During Pregnancy and Risk of Gestational Diabetes Mellitus: A Systematic Review and Meta-Analysis. J Viral Hepat 25(11), 1372-1383 (2018)

24. J. Weng, Y. Li, W. Xu, L. Shi, Q. Zhang et al., Effect of intensive insulin therapy on beta-cell function and glycaemic control in patients with newly diagnosed type 2 diabetes: a multi-centre randomised parallelgroup trial. Lancet 371, 1753-1760 (2008)

25. X. Hou, J. Liu, J. Song, C. Wang, K. Liang et al. (2016) Relationship of hemoglobin A1c with $\beta$ cell function and insulin resistance in newly diagnosed and drug naive type 2 diabetes patients. J Diabetes Res2016:8797316

26. W.J. Zhou, J.J. Jin, Y.H. Wu, K.Y. Gong, J.S. Zhang et al., Explore the occurrence and development of $\beta$ cell dysfunction and insulin resistance according to on the stratification on normal glucose tolerance. Chin J Endocrinol Metab (Chin) 33, 741-744 (2017)

27. W.T. Wang, X.Q. Zhao, G.P. Li, Y.Z. Chen, L. Wang et al., Immune response pattern varies with the natural history of chronic hepatitis B. World J. Gastroenterol. 25(16), 1950-1963 (2019)

28. C. Wu, S.A. Khan, L.J. Peng, H. Li, S.G. Carmella et al., Perturbation of glucose flux in the liver by decreasing F26P2 levels causes hepatic insulin resistance and hyperglycemia. Am JPhysiolEndocrinolMetab 291, E536-E543 (2006)

29. H.Y. Kim, H.K. Cho, S.K. Yoo, J.H. Cheong, Hepatic STAMP2 deceases hepatitis B virus X proteinassociated metabolic deregulation. Exp Mol Med 44, 622-632 (2012) 
30. K. Kim, K.H. Kim, J. Cheong, Hepatitis B virus X protein impairs hepatic insulin signaling through degradation of IRS1 and induction of SOCS3. PLOS ONE 5, e8649 (2010)

\section{Unsectioned Paragraphs}

\section{TABLE}

\section{Figures}
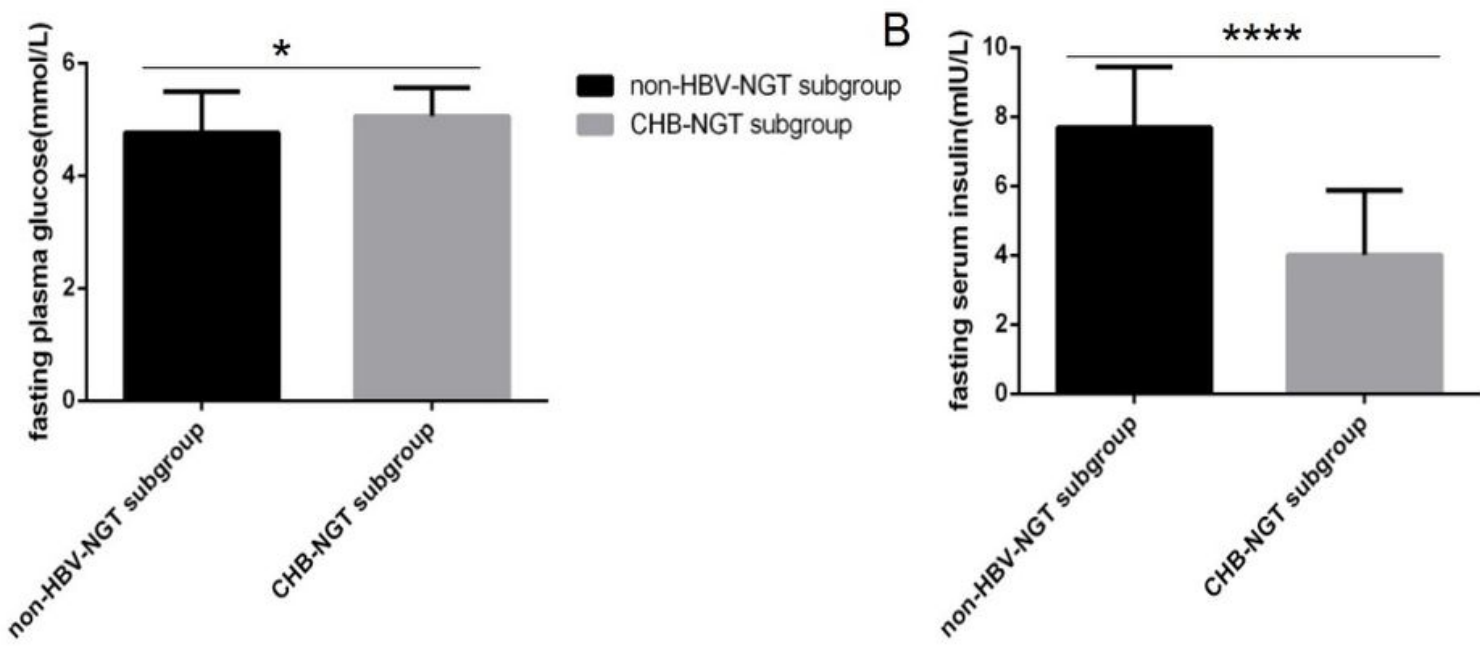

\section{C}

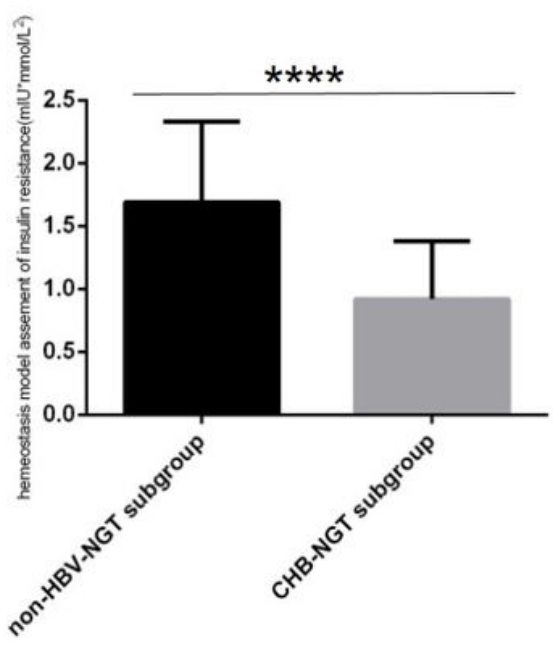

non-HBV-NGT subgroup CHB-NGT subgroup

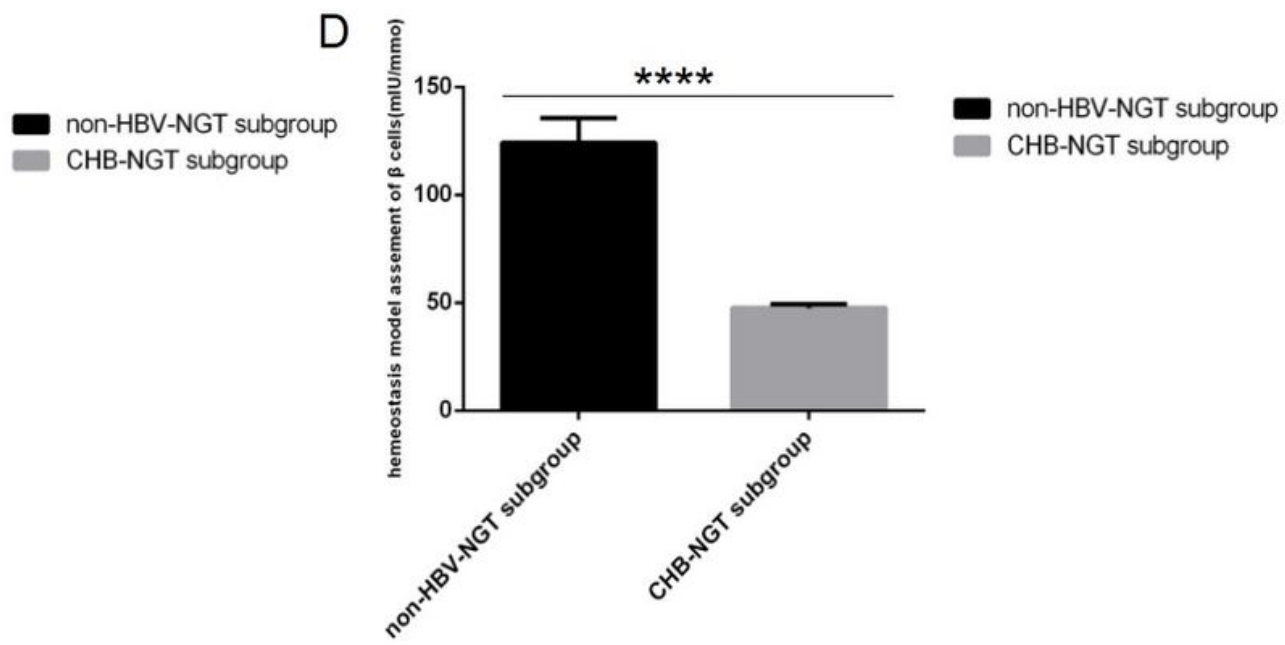

\section{Figure 1}

Comparison of the glucose metabolic parameters between the two groups under NGT condition $(n=100 ; C H B$ group, $n=50$, non-HBV group, $n=50$ ). A. FPG level; B. FINS level; C. HOMA-IR value; D. HOMA- $\beta$ value. Abbreviations: FPG, fasting plasma glucose; FINS, fasting serum insulin; HOMA-IR, homeostasis model assessment of insulin resistance; HOMA- $\beta$, homeostasis model assessment of $\beta$ cell function; NGT, normal glucose tolerance; $\mathrm{CHB}$, cirrhosis hepatitis $\mathrm{B}$; non-HBV, without hepatitis B virus infection. Unmatched t-tests

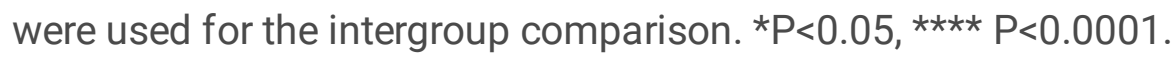



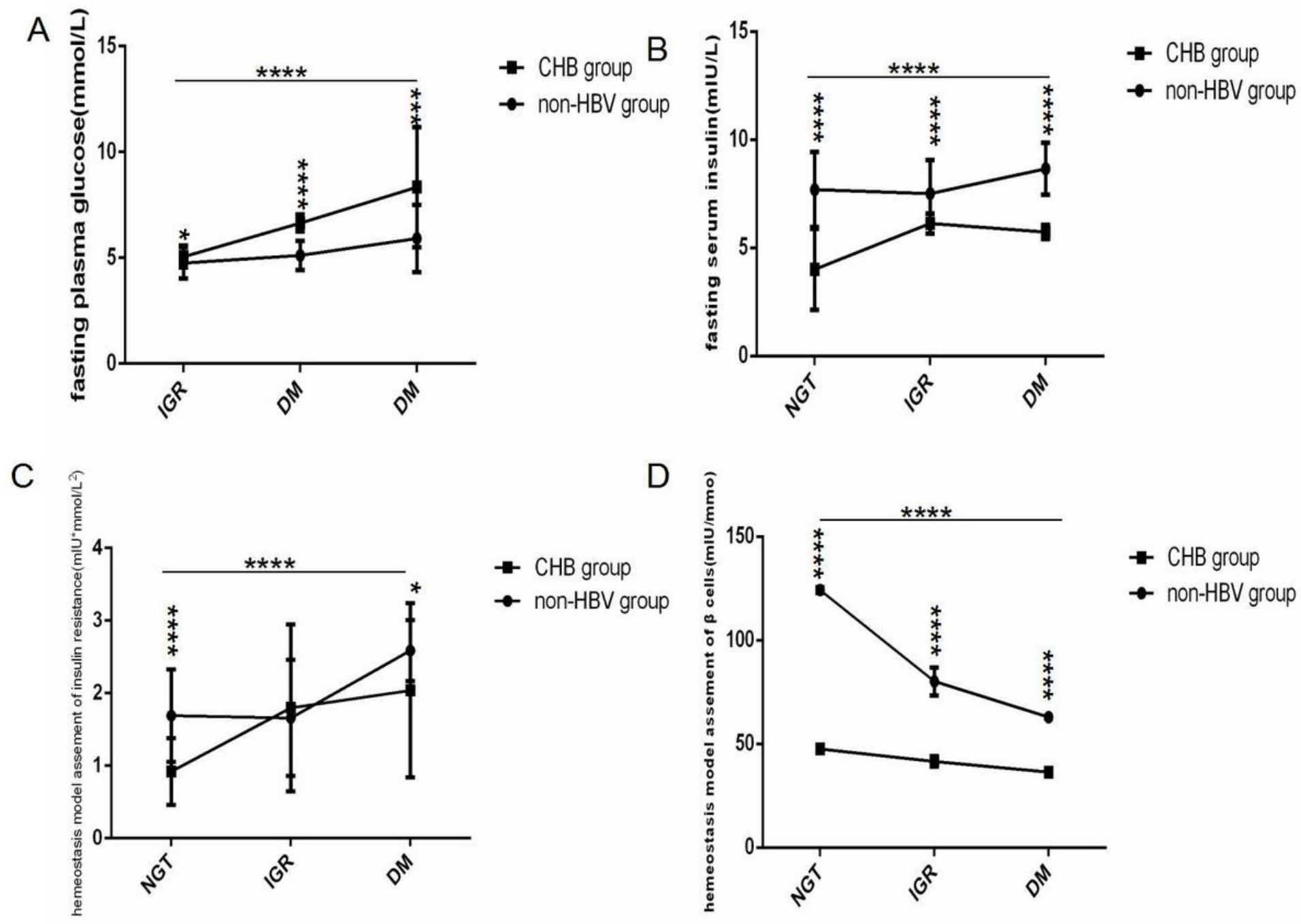

\section{Figure 2}

Comparison of the glucose metabolism parameters between the two groups under NGT, IGR and DM conditions ( $n=220 ; N G T$, IGR, and DM in the two groups, $n=50,30$ and 30, respectively). A. FPG levels; B. FINS levels; C. HOMA-IR values; D. HOMA- $\beta$ values. Abbreviations: FPG, fasting plasma glucose. FINS, fasting serum insulin; HOMA-IR, homeostasis model assessment of insulin resistance; HOMA- $\beta$, homeostasis model assessment of $\beta$ cell function; NGT, normal glucose tolerance; IGR, impaired glucose regulation; DM, diabetes mellitus. Two-way ANOVA was used for the interaction comparison $(A, B, D$, all $P<0.0001 ; C, P<0.01)$. Oneway ANOVA was used for intragroup comparisons $(A, B, C, D$, all $P<0.0001)$. Unmatched t-tests were used for the intergroup comparisons. ${ }^{*} P<0.05, * \star * P<0.001, * \star * P<0.001$. 

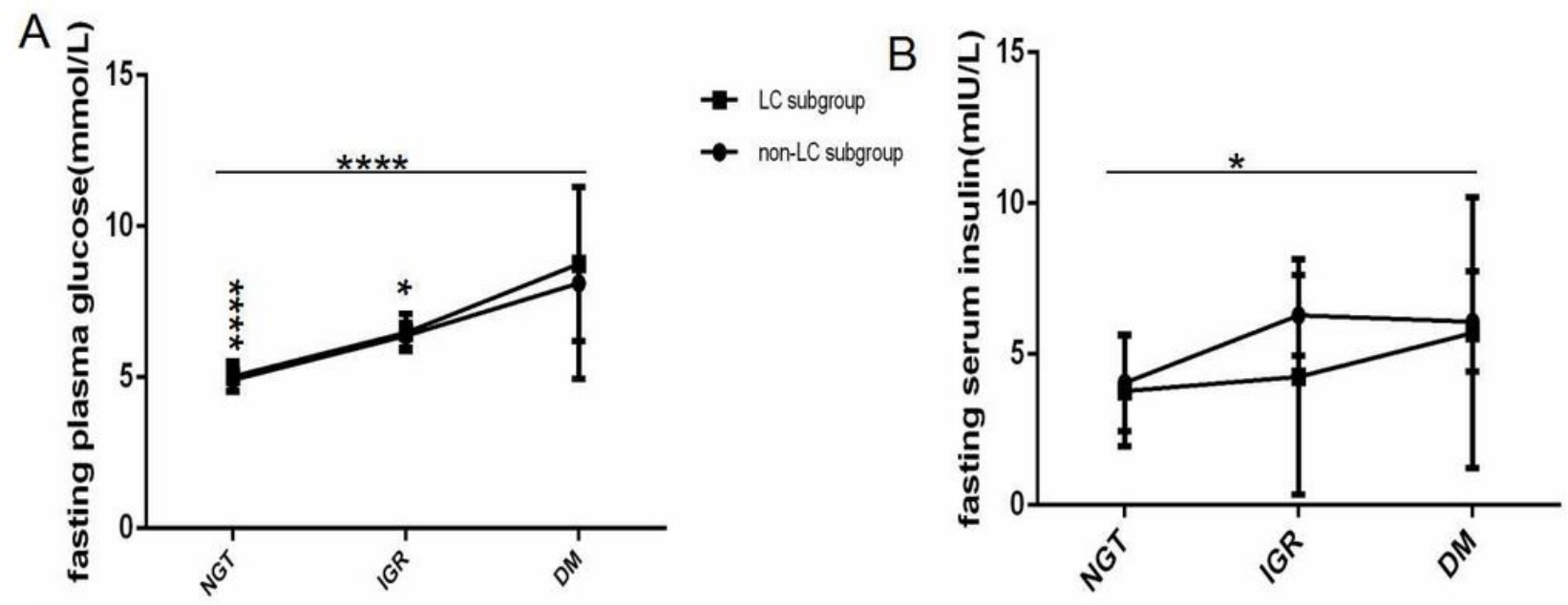

C

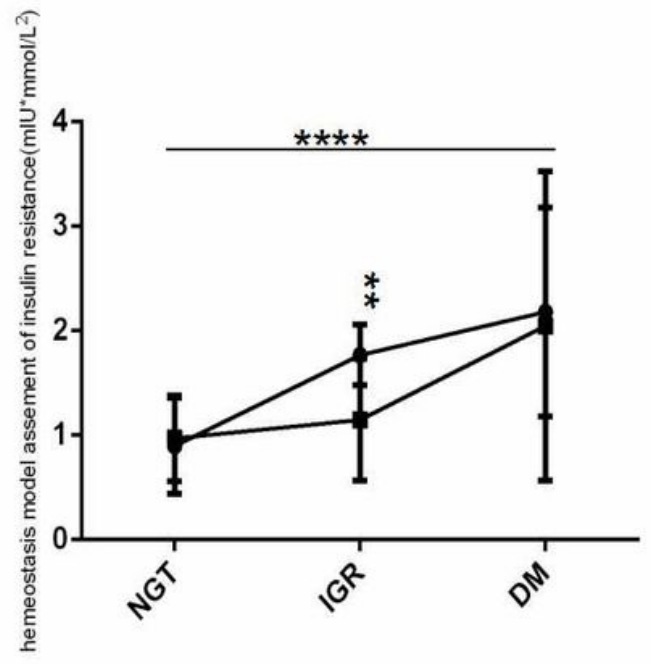

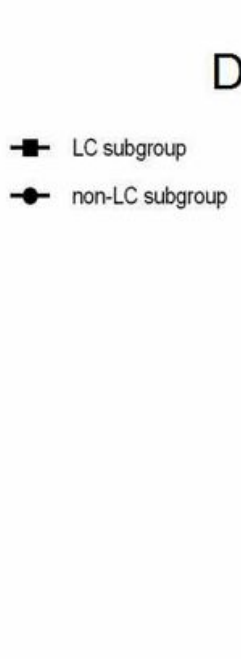

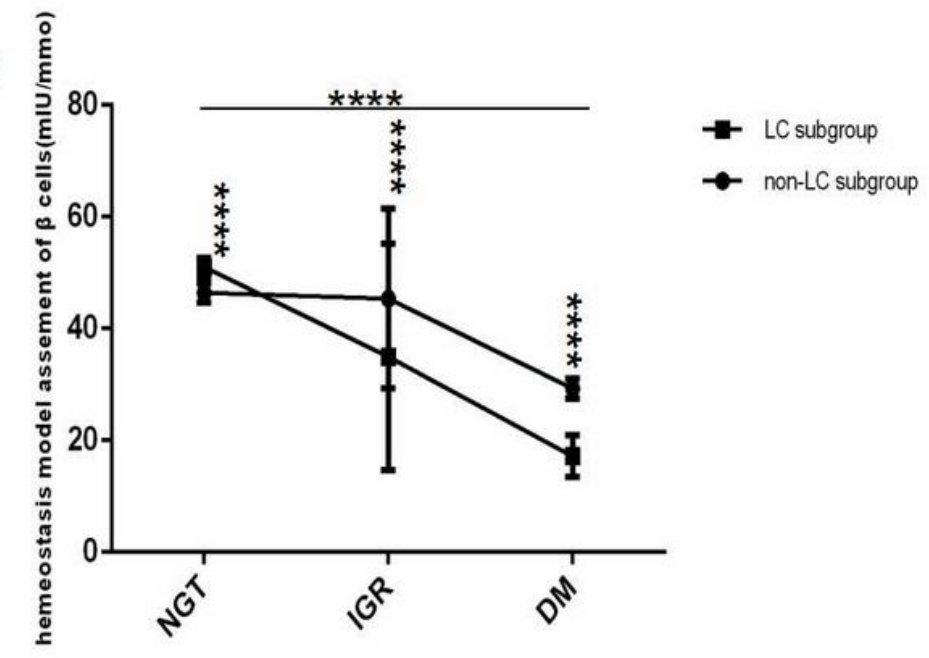

Figure 3

Comparison of glucose metabolism parameters between the non-LC and LC CHB subgroups under NGT, IGR and DM conditions ( $n=110$; NGT, IGR and DM in non-LC subgroup, $n=42,12$ and 15 , respectively; in LC subgroups, $n=8,18$ and 15 , respectively). A. FPG levels; B. FINS levels; C. HOMA-IR values; D. HOMA- $\beta$ values. Abbreviations: FPG, fasting plasma glucose; FINS, fasting serum insulin; HOMA-IR, homeostasis model assessment of insulin resistance; HOMA- $\beta$, homeostasis model assessment of $\beta$ cell function; NGT, normal glucose tolerance; IGR, impaired glucose regulation; DM, diabetes mellitus; $\mathrm{CHB}$, chronic hepatitis $\mathrm{B}$; LC, liver cirrhosis. Two-way ANOVA was used for the interaction comparison ( $A, P<0.05 ; D, P<0.0001)$. One-way ANOVA was used for intragroup comparisons $(A, C, D$, all $P<0.0001 ; B, P<0.05)$. Unmatched t-tests were used for the intergroup comparisons. * $P<0.05,{ }^{\star *} P<0.01,{ }^{\star *} * \mathrm{P}<0.001$. 
A

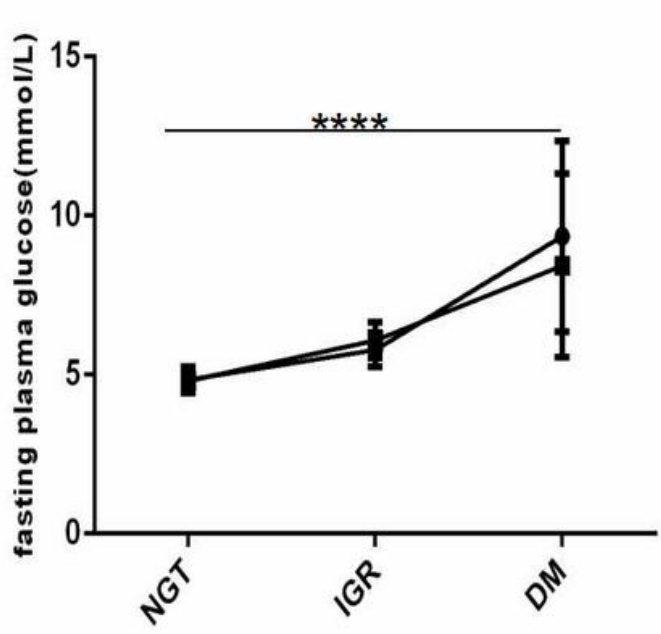

C

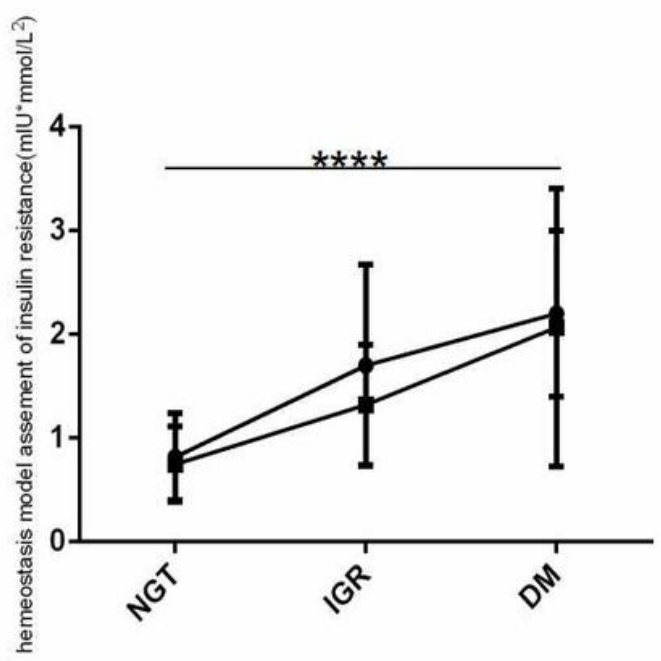

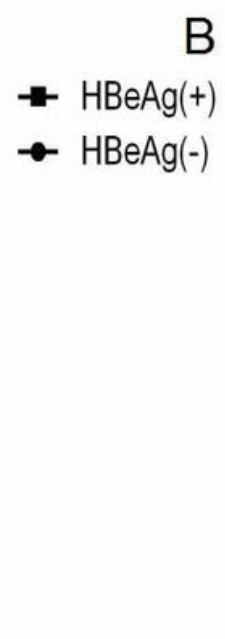

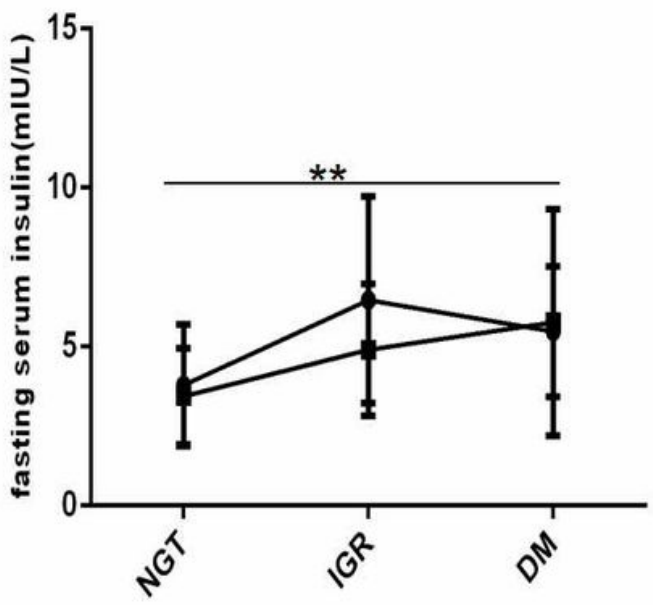

HBeAg(+)

$\rightarrow \mathrm{HBeAg}(-)$

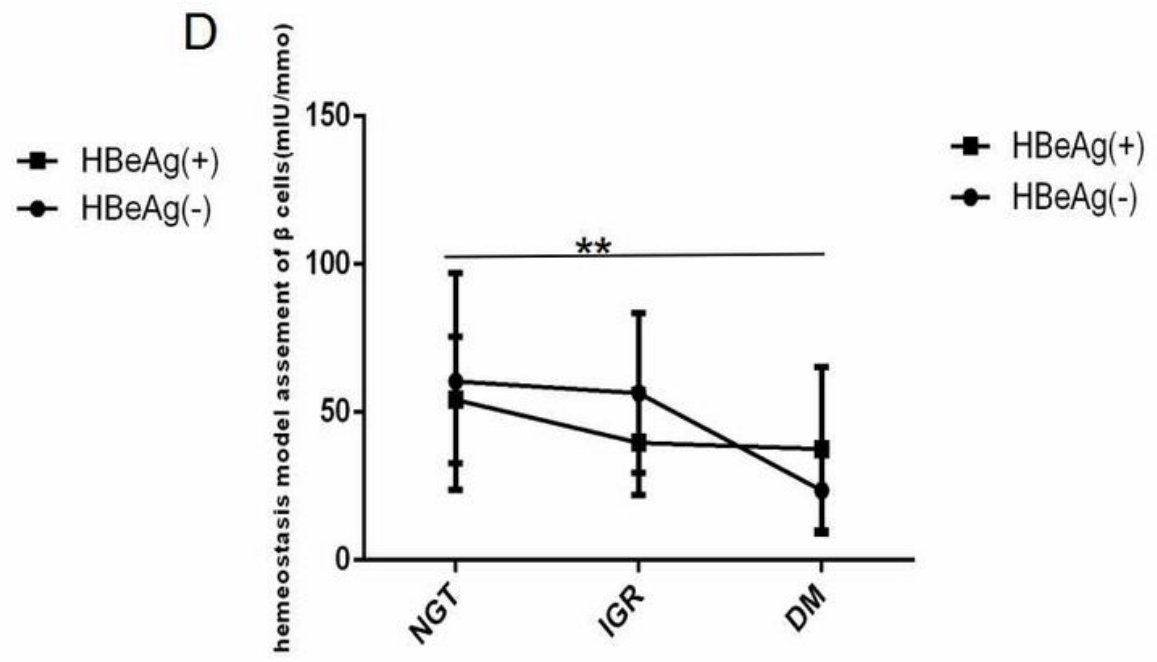

\section{Figure 4}

Comparison of glucose metabolism parameters between the HBeAg (+) and $\mathrm{HBeAg}(-)$ subgroups under NGT, IGR and DM conditions ( $n=110$; NGT, IGR and DM in HBeAg (-) subgroup, $n=24,23$ and 25, respectively; in HBeAg (+) subgroup, $n=26,7$ and 5, respectively). A. FPG levels; B. FINS levels; C. HOMA-IR values; D. HOMA$\beta$ values. Abbreviations: FPG, fasting plasma glucose; FINS, fasting serum insulin; HOMA-IR, homeostasis model assessment of insulin resistance; HOMA- $\beta$, homeostasis model assessment of $\beta$ cell function; NGT, normal glucose tolerance; IGR, impaired glucose regulation; DM, diabetes mellitus; $\mathrm{HBeAg}(-)$, hepatitis $B$ envelope antigen negative; $\mathrm{HBeAg}(+)$, hepatitis $B$ envelope antigen positive. Two-way ANOVA was used for interaction comparisons $(A, B, C, D$, all $P>0.05)$. One-way ANOVA was used for intragroup comparisons ( $A, C$, all $P<0.0001 ; B, D$, all $P<0.01)$. Unmatched t-tests were used for the intergroup comparisons $(A, B, C, D$, all $P>0.05)$. 